\title{
Diagnostic Studies for Excretion Be - Star Disc Evolution in Be/X-Ray Binary Systems Utilizing SALT Spectroscopy *
}

\author{
A.F. Rajoelimanana ${ }^{1 \dagger}$, P.J. Meintjes ${ }^{1}$, P. A. Charles ${ }^{2,3}$ \\ ${ }^{1}$ Department of Physics, University of the Free State, PO Box 339, Bloemfontein 9300, SA \\ ${ }^{2}$ School of Physics and Astronomy, Southampton University, Southampton SO17 1BJ \\ ${ }^{3}$ Astrophysics, Cosmology and Gravity Centre, Department of Astronomy, University of Cape \\ Town, Rondebosch 7701, South Africa
}

E-mail: rajoelimananaa@ufs.ac.za

\begin{abstract}
Our study of the long-term superorbital variability in Be X-ray binaries using 22 yrs light curve from MACHO and OGLE databases, has revealed 200-3000d quasi-period variations in almost all of them with amplitudes ranging up to $1.2 \mathrm{mag}$. These variations are related to the formation and dissipation of the Be star circumstellar disc. We present the preliminary results of optical spectroscopic observations of a sample of Magellanic Clouds Be/X-ray binaries (BeX) taken throughout their superorbital cycle. These observations were carried out with the Southern African Large Telescope (SALT) using the Robert Stobie Spectrograph (RSS) in long-slit mode. For each observing night, we obtained both wide wavelength and high-resolution spectra. This allows us to follow the evolution of the size and structure of the Be equatorial disc and to separate the various emitting components, in particular the spectrum of the Be disc. We used the high-resolution spectra for spectral classification of the underlying B star and determination of the rotational and radial velocities.
\end{abstract}

Frontier Research in Astrophysics - II

23-28 May 2016

Mondello (Palermo), Italy

* Based on observations made with the Southern African Large Telescope (SALT).

$\dagger$ Speaker. 


\section{Introduction}

The Magellanic Clouds, particularly the Small Magellanic Cloud (SMC), are well-known for hosting a large number of high-mass X-ray binaries (HMXBs) when compared to our Galaxy. The most recent catalogue of HMXBs in the SMC by [1] listed 121 confirmed HMXBs, in 63 of them $\mathrm{X}$-ray pulsations were detected. The majority of these HMXBs are BeX systems consisting of a neutron star (NS), usually an X-ray pulsar, orbiting an early-type rapidly rotating Be star in a wide $\left(P_{\text {orb }} \sim 10-300 \mathrm{~d}\right)$ and very eccentric orbit (e $\geq 0.3$ ). In BeX systems, when the circumstellar disc around the Be star is sufficiently extended, the neutron star (NS) can interact with it during each periastron passage, causing activity (sometimes a luminous X-ray outburst) that may be observed over a wide range of wavelengths (optical, IR, X-ray...). A detailed review of the observational properties of BeX systems may be found in [2,3]. The very long orbital periods in these systems have made detailed phase-resolved spectroscopy extremely difficult (if not impossible), and only occasional spectra have been obtained of most BeX systems (usually as part of their identification as an X-ray source). Here we report on the optical spectroscopic monitoring of a sample of Magellanic Clouds BeX systems (see Table 1), undertaken with the Southern African Large Telescope (SALT).

\section{Long-term superorbital variation}

Apart from the orbital modulations, seen as regular outbursts, BeX systems also show much longer superorbital variations on timescales of hundreds to thousands of days. The Large Magellanic Cloud (LMC) BeX source A0538-66 was the first reported to show this behavior $[4,5]$ thanks to the MACHO long-term monitoring project. This motivated us to conduct a systematic study of long-term optical variability of BeX systems in the SMC using the combined 22 yrs light curve from MACHO and OGLE databases [6]. We found that almost all of these BeX systems exhibit a quasi-periodic superorbital variation on timescales of $200-3000 \mathrm{~d}$. These variations are believed to be related to the formation and dissipation of the Be star circumstellar disk. However, the physical mechanisms controlling these discs are not well understood.

Moreover, their MACHO color $(V-R)$ varies with their optical brightness. For a Be star seen at a low inclination angle (e.g SXP6.85), the source is brighter when redder as the disc adds cooler (redder) light to that of the Be star. However, if seen edge-on (e.g A0538-66), as the equatorial disc forms it masks part of the hotter Be star, making the source appear fainter when redder. Examples of long-term light curves and MACHO color variation of two BeX systems, SXP6.85 (a low inclination system) and A0538-66 (a high inclination system), are shown in Figure 1. Both of them display high-amplitude superorbital variations with a period of $621 \mathrm{~d}$ and $420 \mathrm{~d}$ respectively, however, the behaviour of their MACHO color is different.

\section{SALT observations}

We have selected a sample of Magellanic BeX systems from [6] which display high amplitude quasi-period long-term variations in their light curves. All sources in the sample (see Table 1) 

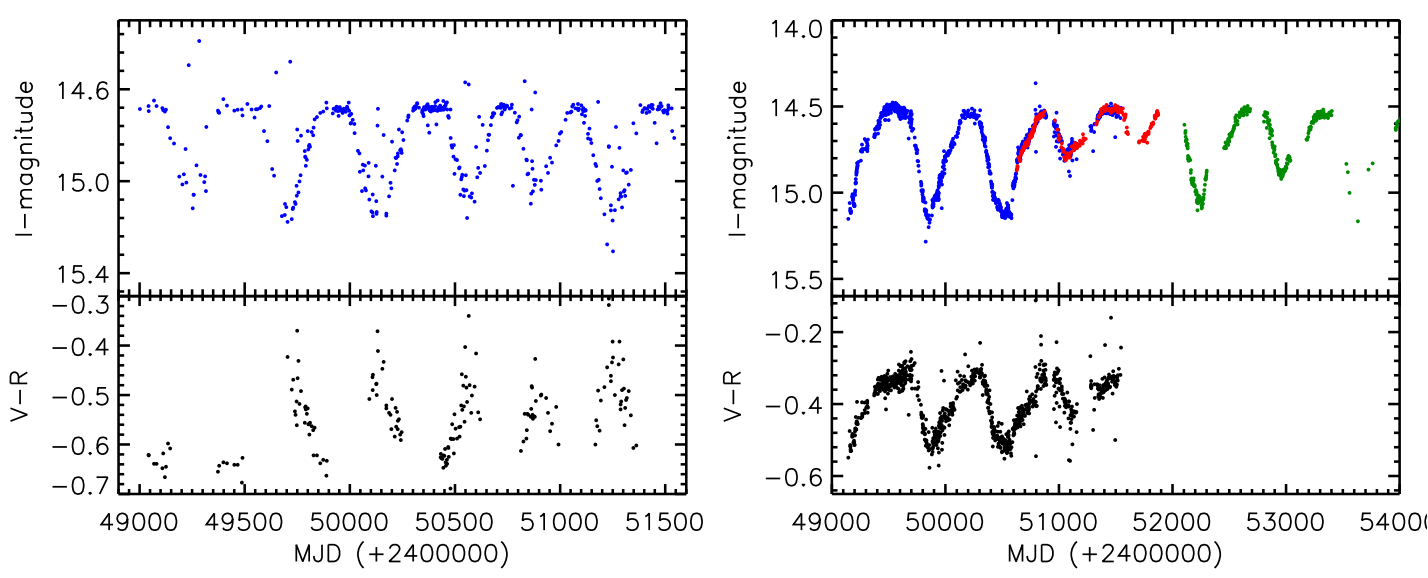

Figure 1: Long-term light curves (top) and MACHO color variations(bottom) of the high inclination system A0538-66 (left) and the low inclination system SXP6.85 (right). Note the difference in the behaviour of their MACHO color.

Table 1: List of Magellanic Clouds BeX systems in our sample.

\begin{tabular}{lccccc}
\hline \hline Short ID & RA & Dec & V mag & $P_{\text {orb }}$ & $P_{\text {sup }}$ \\
\hline SXP2.37 & $00: 54: 33$ & $-73: 41: 01.3$ & 16.3 & 18.58 & - \\
SXP2.76 & $00: 59: 13$ & $-71: 38: 44.9$ & 14 & 82.37 & 2800 \\
SXP6.85 & $01: 02: 53$ & $-72: 44: 35.1$ & 14.5 & 110.0 & 621 \\
SXP7.92 & $00: 57: 59$ & $-72: 22: 29.2$ & 13.9 & 36.41 & 397 \\
SXP8.80 & $00: 51: 53$ & $-72: 31: 48.6$ & 14.8 & 28.51 & 1798 \\
SXP9.13 & $00: 49: 14$ & $-73: 11: 37.8$ & 16.5 & 80.10 & 1886 \\
SXP15.3 & $00: 52: 14$ & $-73: 19: 18.8$ & 14.6 & 74.51 & 1515 \\
SXP22.1 & $01: 17: 40$ & $-73: 30: 50.6$ & 14.1 & 75.97 & - \\
SXP59.0 & $00: 54: 56$ & $-72: 26: 47.8$ & 15.2 & 62.10 & - \\
SXP202A & $00: 59: 21$ & $-72: 23: 17.4$ & 14.8 & 71.98 & 1220 \\
SXP202B & $00: 59: 29$ & $-72: 37: 04.2$ & 15.6 & 224.0 & 3000 \\
SXP293 & $00: 58: 13$ & $-72: 30: 48.8$ & 14.9 & 59.77 & - \\
SXP645 & $00: 55: 35$ & $-72: 29: 06.6$ & 14.6 & 135.3 & 2857 \\
1A0538-66 & $05: 35: 41$ & $-66: 51: 53.7$ & 14.9 & 16.64 & 420 \\
\hline
\end{tabular}

were observed in a regular and systematic way throughout their superorbital phase. We used the on-going OGLE-IV observations ${ }^{1}$ to predict the superorbital phase of our SALT observations.

The spectroscopic observations were carried out with the Robert Stobie Spectrograph (RSS) [7, 8] on the Southern African Large Telescope (SALT) [9, 10] at SAAO, Sutherland. For each observing night, we obtained both a low-resolution wide wavelength (3200 - $9000 \AA)$ and highresolution spectrum. These spectra were used to determine the physical properties of this BeX source, investigate the formation and dissipation of the equatorial disc, and the interaction of the

\footnotetext{
${ }^{1}$ real-time light curves are available at: http://ogle.astrouw.edu.pl/ogle4/xrom/xrom.html
} 
NS with the Be equatorial disc. High-resolution spectra are required to accurately classify the hot star spectral type that are essential for modelling the disc properties.

The primary reduction of the data was done with the IRAF package PYSALT [11] which include overscan, gain and cross-talk correction. Wavelength calibration, sky subtraction and flux calibration were performed using the standard IRAF software tasks in TWODSPEC. One-dimensional spectra were then extracted using the IRAF task APALL. Because of the design of the SALT telescope, an absolute flux calibration is not possible, however, the spectral shape can still be recovered by applying a relative flux correction derived from the observed spectrophotometric standard.

\subsection{Broad-band spectrum}

To obtain wide wavelength range spectra, we have combined two sets of spectra obtained from the two different settings of the 900 lines $\mathrm{mm}^{-1}$ grating (PG0900), yielding coverage from $\sim 3100-9000 \AA$ at a dispersion of $\sim 1.0 \AA$ A.pixel ${ }^{-1}$ and a spectral resolution of $190 \mathrm{~km} \mathrm{~s}^{-1}$ $(\sim 4.0 \AA)$ at $\mathrm{H} \alpha$. Be stars are characterized by the presence of an infrared excess and emission spectral lines in their spectra, originating from an extended circumstellar disc around the B star. For a low inclination system, the contribution from the disc is (usually) negligible during optical minima (disc-less phase); therefore spectra taken around this time will give us direct information about the state of the naked B star. On the other hand, the spectrum taken at optical maxima will contain information about the disc. By comparing and modelling these two observations, we aimed to separate the components due to the (hot) Be primary, the possible X-ray irradiated disc, and the (cooler) equatorial disc. Examples of wide wavelength spectra of BeX systems taken during optical low and high state are shown in Figure 2. It is evident that the spectrum shows a very strong $\mathrm{H} \alpha$ emission line at optical high state and $\mathrm{H} \alpha$ absorption line during optical low states.

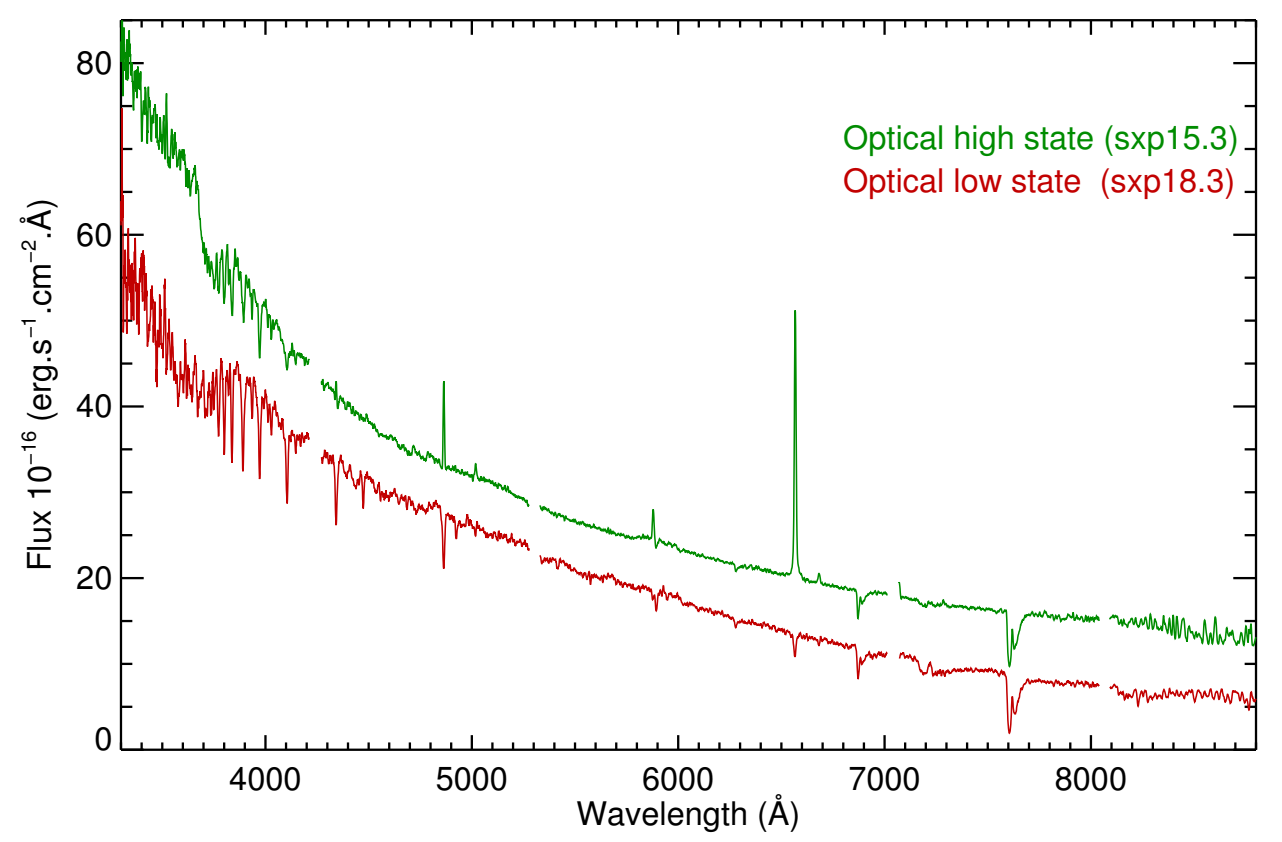

Figure 2: Broad-band spectra of BeX system taken during optical low (red) and high (green) state. The gaps in the spectra are CCD gaps. 


\subsection{High-resolution spectra}

The High-resolution spectra were used to measure the rotational and radial velocity of the Be star and provide a more accurate spectral classification. As a result of the low metallicity of the Magellanic Clouds, the metal lines in B star spectra are very weak, making the standard spectra classification, based on metal-helium ratios, difficult $[12,13,14]$. We used, therefore, the classification criteria presented in [12] for B-type stars in the SMC which is based on the presence of the He I and He II $(\lambda 4200, \lambda 4541, \lambda 4686)$ line for the earliest subtypes and on the metal line strengths for the latest subtype. Examples of high-resolution spectra of some BeX systems from our sample are shown in Fig. 3.

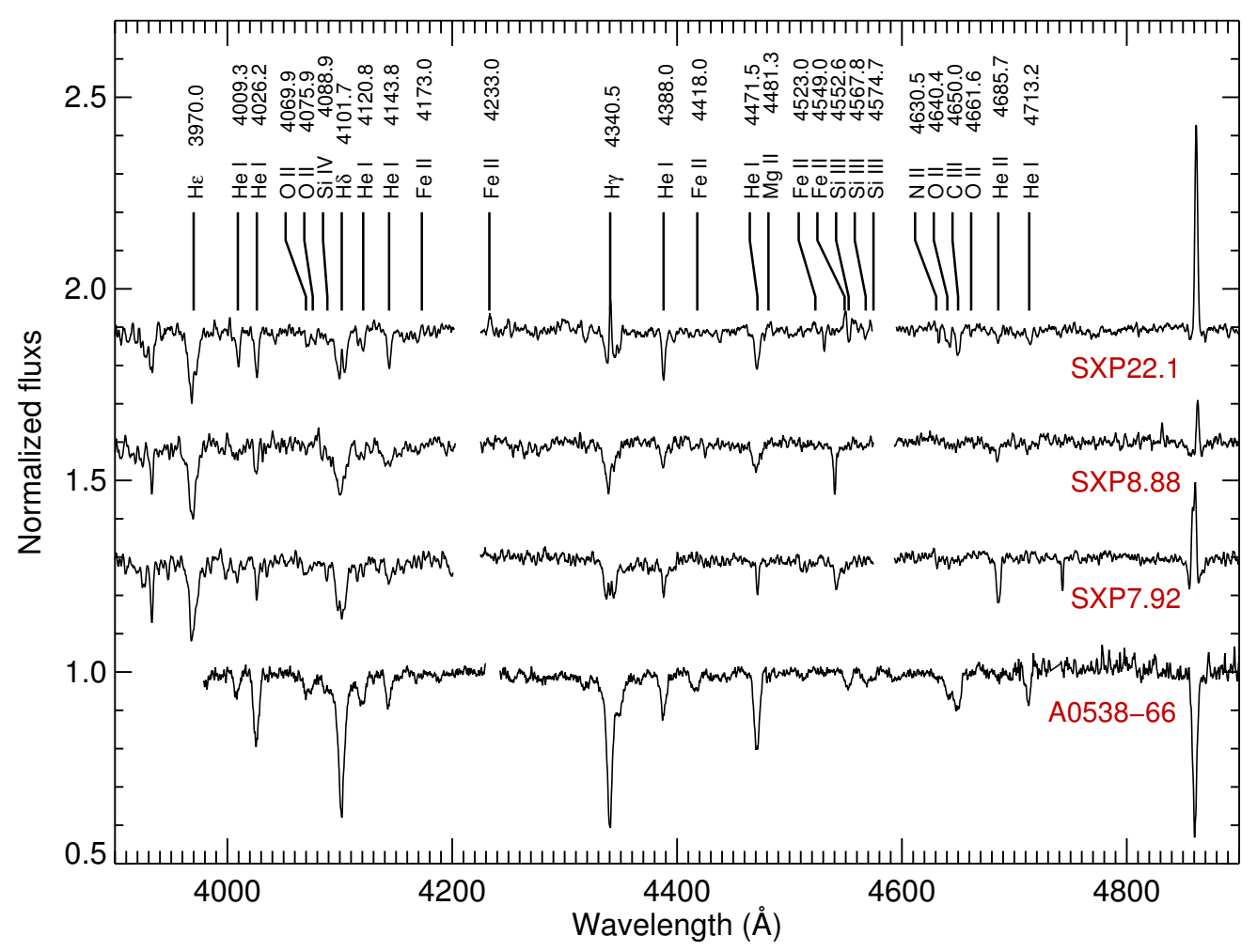

Figure 3: The observed SALT high-resolution spectra of some BeX systems from our sample. The gaps in the spectra are CCD gaps.

The rotational velocities were estimated from profiles of some He I absorption lines ( $\lambda 4026$, $\lambda 4143, \lambda 4387$ and $\lambda 4471)$. The He I lines are strong throughout the B spectral sequence, and are less influenced by emission from the circumstellar disk than the Balmer lines. The measured Full Width Half Maximum (FWHM) of these lines were converted to rotational velocities using the relations given in [15]. For each source, we used the spectra with the weakest $\mathrm{H} \alpha$ line to reduce the contamination from the circumstellar disc. Another way of estimating the rotational velocity is by comparing the observed spectra with synthetic spectra rotationally broadened from 50 - 500 $\mathrm{km} \mathrm{s}^{-1}$ in steps of $10 \mathrm{~km} \mathrm{~s}^{-1}$. We used the BSTAR2006 grids of a model spectrum from [16]. The model spectra were also instrumentally broadened with Gaussian with FWHM of the instrument. We have also obtained the radial velocity curves of the He I lines, which are less affected by the disc 
emission, by cross-correlating each individual spectra with this instrumentally broadened synthetic spectrum.

\section{3 $\mathrm{H} \alpha$ line profile}

The Balmer emission lines in Be star originate from the circumstellar discs. Therefore, longterm monitoring of the Balmer emission lines, especially the $\mathrm{H} \alpha$, should reveal the long-term changes in the size and structure of the Be equatorial disc. Our long-term optical spectra show a wide range of variability over both short and long temporal scales in their $\mathrm{H} \alpha$ emission line. A0538-66 is one example of the $\mathrm{BeX}$ system showing this behaviour. Its $\mathrm{H} \alpha$ line shows a variety of profile shapes which include symmetric and asymmetric shell profiles, asymmetric double-peaked profiles, and single-peaked profiles. Interestingly, the single-peaked and double-peaked profiles are only seen in the spectra taken at periastron and the symmetric shell profiles in the spectra taken far from the periastron. This means that far from periastron we have an unperturbed equatorial disc viewed nearly edge-on but near periastron, the disc is distorted by the NS gravity. Moreover, the double-peaked profiles are asymmetric with the red component much stronger than the blue components $(\mathrm{V}<\mathrm{R})$. This is consistent with our orbital solution $\omega=183^{\circ}$ and $e=0.72$ (the NS is moving away from us near periastron) derived from our radial velocity curve. Figure 4 shows the evolution of the $\mathrm{H} \alpha$ line profile of A0538-66 sorted in time sequence (left) and by orbital phase (right). We note that phase 0 is defined as the time when the outburst reaches its maximum brightness and periastron is at phase 0.04 .

\section{Summary}

We have used SALT/RSS to conduct an optical spectroscopic monitoring of a sample of Magellanic Clouds BeX system. SALT, with its Q-scheduled operational mode and wide wavelength coverage, have yielded promising results in order to follow the global changes in the structure of the Be equatorial disc. These changes in the strength and shape of the line profiles are associated with both the formation and dissipation of the Be circumstellar disc and its interaction with the NS during periastron passage. Our high-resolution spectra are of sufficient resolution and S/N to accurately classify its optical counterpart and to obtain a measurement of the rotational and radial velocities.

\section{Acknowledgments}

Some of the observations reported in this paper were obtained with the Southern African Large Telescope (SALT) under program 2011-3-RSA_UKSC-008 (PI: Charles), 2011-3-RSA_UKSC007, 2012-1-RSA_UKSC-005, 2012-1-RSA_UKSC-006 and 2012-2-RSA_UKSC-003 (PI: Rajoelimanana). This paper utilizes public domain data obtained by the MACHO Project, jointly funded by the US Department of Energy through the University of California, Lawrence Livermore National Laboratory under contract No. W-7405-Eng-48, by the National Science Foundation through the Center for Particle Astrophysics of the University of California under cooperative agreement AST-8809616, and by the Mount Stromlo and Siding Spring Observatory, part of the Australian National University. 

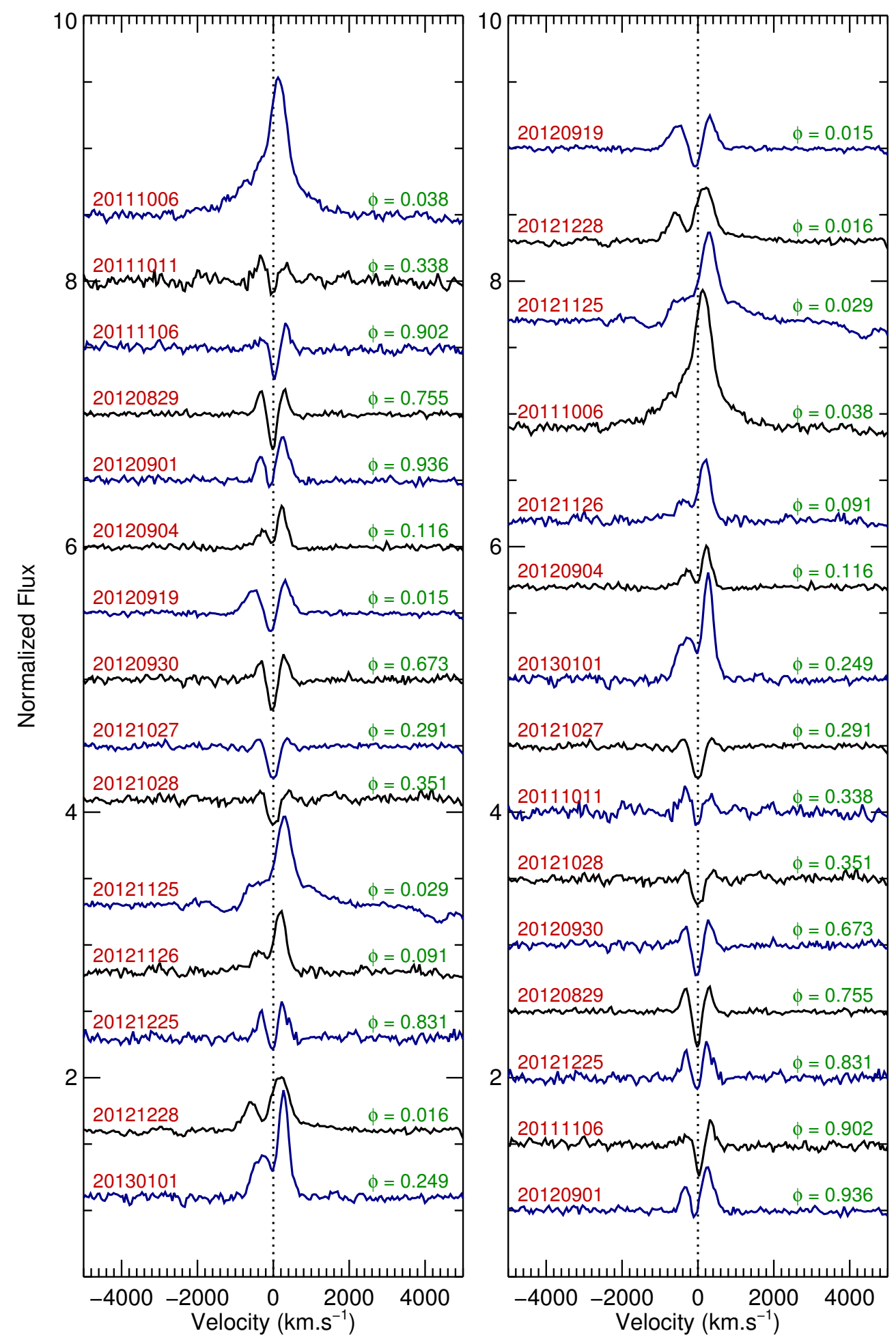

Figure 4: Evolution of the $\mathrm{H} \alpha$ line profile sorted in time sequence (left) and by orbital phase (right). The dotted lines indicates the rest velocity. Phase 0 is defined as the time when the outburst reaches its maximum brightness. Note the change in the line profiles from double-peaked to singled-peak at periastron $(\phi=0.038)$ and symmetric shell profile far from periastron. 


\section{References}

[1] Haberl F., Sturm R., 2016, A\&A, 586, A81

[2] Reig P., 2011, Ap\&SS, 332, 1

[3] Coe M. J., Kirk J., 2015, MNRAS, 452, 969

[4] Alcock C., Allsman R. A., Alves D. R., et al., 2001, MNRAS, 321, 678

[5] McGowan K. E., Charles P. A., 2003, MNRAS, 339, 748

[6] Rajoelimanana A. F., Charles P. A., Udalski A., 2011, MNRAS, 413, 1600

[7] Burgh E. B., Nordsieck K. H., Kobulnicky H. A., Williams T. B., O’Donoghue D., Smith M. P., Percival J. W., 2003, SPIE, 4841, 1463

[8] Kobulnicky H. A., Nordsieck K. H., Burgh E. B., Smith M. P., Percival J. W., Williams T. B., O’Donoghue D., 2003, SPIE, 4841, 1634

[9] Buckley D. A. H., Swart G. P., Meiring J. G., 2006, SPIE, 6267, 62670Z

[10] O’Donoghue D., Buckley D. A. H., Balona L. A., et al., 2006, MNRAS, 372, 151

[11] Crawford S. M., Still M., Schellart P., et al., 2010, SPIE, 7737, 773725

[12] Evans C. J., Howarth I. D., Irwin M. J., Burnley A. W., Harries T. J., 2004, MNRAS, 353, 601

[13] McBride V. A., Coe M. J., Negueruela I., Schurch M. P. E., McGowan K. E., 2008, MNRAS, 388, 1198

[14] Antoniou V., Hatzidimitriou D., Zezas A., Reig P., 2009, ApJ, 707, 1080

[15] Steele I. A., Negueruela I., Clark J. S., 1999, A\&AS, 137, 147

[16] Lanz T., Hubeny I., 2007, ApJS, 169, 83 